\title{
Iris positioning technique by using face symmetric measurement tool on the custom ocular prosthesis: a case report
}

\author{
${ }^{1}$ Femy Rilinda, ${ }^{2}$ Haslinda Z. Tamin, ${ }^{2}$ Putri Welda Utami Ritonga \\ ${ }^{1}$ Postgraduate Program in Prosthodontics \\ ${ }^{2}$ Department of Prosthodontics \\ Faculty of Dentistry, Universitas Sumatera Utara \\ Medan, Indonesia \\ Corresponding author: Femy Rilinda, E-mail: femy_ling2@yahoo.co.id
}

\begin{abstract}
Loss of eye can have a major psychological impact on the patient. To overcome this problem, an ocular prosthetic musthave been fabricated similar with the natural eye. Various techniques are documented to determine the symmetrical bilateral iris position; these methods can be subjective and have no measurement guidelines. A20-year-old male patient came to the Prosthodontic Clinic of Dental Hospital Universitas Sumatera Utara with the diagnosis is post enucleation socket syndrome. Determination of the iris position using the face symmetric measurement-tool by focus to the vertical and horizontal lines of the facial symmetry guide. Confirm the alignment of the vertical lines by connecting the trichion-glabella-subnasal-gnathion guide points so that they vertically divide the two sides of the face, then the two horizontal lines by connecting the left-right cheilon with the mesial-distal canthus of the right and left eyes. This tool is like a face mask with vertical and horizontal guidelines connecting the symmetrical guide points of the face accompanied by centimeters and millimetres making it easy to measure distances and sizes of the iris symmetrically. The use of this tool has givengood results in determining the position of the iris because its simplicity and can see facial symmetry.
\end{abstract}

Keywords: custom ocular prosthesis, iris position, face symmetric measurement tool, face symmetry

This title has been presented in The 12th Biennial Congress of Asian Academy of Prosthodontics, 21 August 2021

\section{INTRODUCTION}

Loss of the eyeball is generally associated with congenital defects, trauma, or pathological abnormalities that may require surgical intervention in removal of the eyeball. ${ }^{1}$ Surgical procedures in this case may require some stages such as orbital evisceration, enucleation or extenation. ${ }^{2}$ Loss of the eyeball can have a major psychological impact.Patients with maxillofacial defects often withdraw, especially on the eye area, resulting in disabling offunctional andsocial activities. Toovercome this problem, an ocular prosthesis can be made that aims to imitate the color, contour, size and orientation of the eye from the existing eye so that it can provide cosmetics eye prostheses. ${ }^{1-3}$

Prosthetic replacement of a missing eye presents many challenges, one of them is determining the accurate size and position of the iris. ${ }^{3-7} \mathrm{An}$ ocularprostheses mustbeaccurate in terms of size and symmetrical iris position, so will give effects such as natural eye duplication. ${ }^{2}$ Various methods, techniques and concepts were documented fordetermining bilaterally symmetrical iris positions in the fabrication of ocular prostheses. ${ }^{4} \mathrm{McArth} u r$ used a method for positioning the iris using an ocular locator and a fixed caliperwith respect to the mediolateral and superior-inferior planes. ${ }^{2,4}$ Robert used a pupilometer. Guttal uses a grid graph. ${ }^{1}$ Benson suggests a visual assessment because position- ing the iris is a sensitive procedure. However, visual assessment and similar techniques are subjective, and neither the grid chart nor the ocular locator technique is well stabilized and cannot be used in patients with facial asymmetry.

Face symmetric measurement tool is a face mask with vertical and horizontal line guides accompanied by a centimeter-millimeter sizethat can connect several reference points. This instrument can effectively measure iris and pupil size as well as right and left binocular iris distance. Theaim of this case report is to describe a simple technique to accurately determine the symmetrical position of the iris in the fabrication of ocular prostheses.

\section{CASE}

A20-year-old male patient came to the Prosthodontics Clinic of the USU General Hospital to have his right ocular prostheses. From the anamnesis, it was known that the etiology of eye losing due to a shot from an air rifle in his eyelids when hewas 9 years old by eye surgery at Pirngadi Hospital, the ophthalmologist gave a manufacturermade ocular and was only instructed not to do too much activity. About 1 month later, the patient lost his ocular prosthese and he has never worn ocular prostheses again due to limited economy.

Objective examination showed that the patient's righteye has been enucleated, healthyorbital 
socketmucosa,superiorsulcus deepening, ptosis, weakness of lower eyelid closure and sufficient space for ocular prosthesis (Fig.1). This condition was diagnosed as post-enucleation socket syndrome. It was decided to make a custom ocular prosthesis.

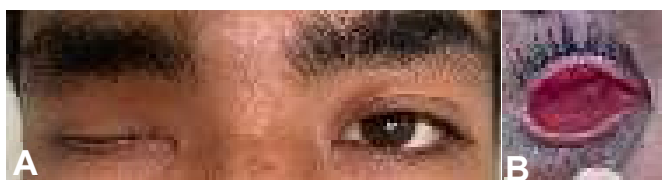

Figure 1A Condition of the eye profile; B eye socket

\section{MANAGEMENT}

Case management began with anatomical impression of the eye socket using a mold made of self-cured acrylic molded with thumb and index fingers attached to a disposable syringe (Fig.2A). Irreversible hydrocolloid impression material (alginate) was injected with the syringe position perpendicular to the eye socket until the impression material came out slightly through the tray hole (Fig.2B).

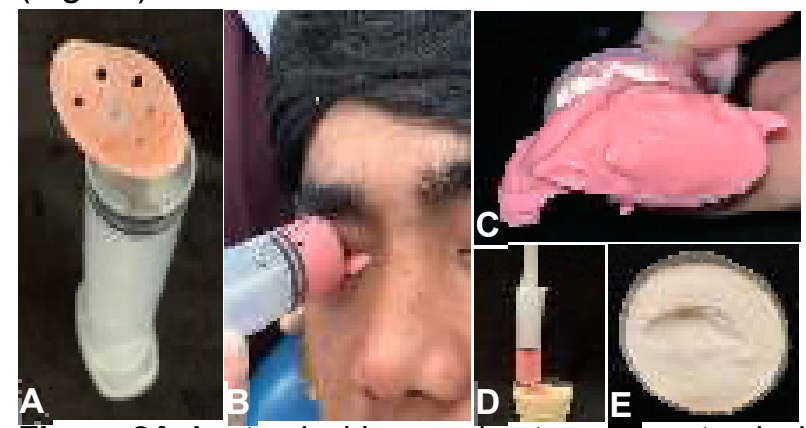

Figure 2A Anatomical impression tray; $\mathbf{B}$ anatomical impression of the socket; C anatomical impression; D the anatomical impression was entered in a container of type IV dental stone; $\mathbf{E}$ anatomical cast of the socket.

Theanatomical impression (Fig.2C)wasentered in a container containing type IV dental stone in an uprightposition(Fig.2D)sothatananatomical cast was obtained and marked the mesial, distal, superior and inferior sections (Fig.2E).

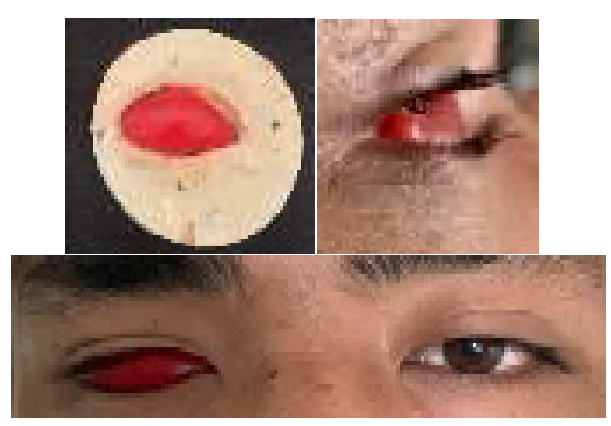

Figure 3A Anatomical scleral wax pattern; B passen anatomical scleral wax pattern seen from the side view; C from the front view.
Then the anatomical cast was filled with a wax patternand shaped like a dome with a convex point at the center apex of the anatomical scleral wax pattern (Fig.3A). The wax pattern was tried in the patient by observing the convex point equal to the left eye (Fig. 3B,C). Then the wax pattern is given three signs, namely the convex point, vertical and horizontal lines as aguide for releasing the final impression tray which will later be made a putty index.

The final impression tray is made from a putty indexmold with self-cure acrylic material (Fig.4A) and a straw as a connector with the tip of the PVS light body impression material (Fig.4B).

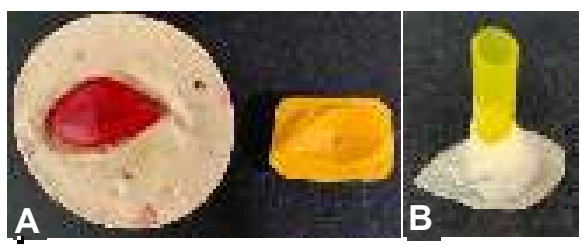

Figure 4A The putty index of the scleral wax convexity; B the final impression tray

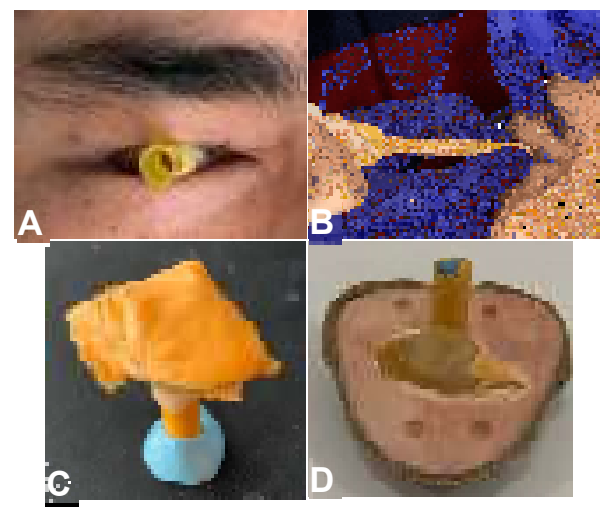

Figure 5A Try in the final impression tray; $\mathbf{B}$ final impression; $\mathbf{C}$ results of final impression; $\mathbf{D}$ entering the mold into the cuvette

The final impression tray was tried in the patient by seeing the straw as a convex top, vertical and horizontal lines are in the middle of the socket while the patient is instructed to activate the eyemovement of opening, closing the eyes, looking left, right, up and down to see if the edge of the final impression tray is at the margin intaglio eye socket (Fig.5A). Impression is carried out by injection of the impression material previously then connecting it to a straw until the socket is full, the tip is removed and the patient activates eye movements (Fig.5B).

The surface of the final impression (Fig.5C) was entered in a cuvette containing type IV dental stone and several key holes were made (Fig.5D). After hardening, the surface is coated with vaseline and refilled to cover the entire surface of the mold, wait for it to harden. 
The final cast was opened, the impression material was removed (Fig.6A) and filled with liquid wax from the hole formed from the previous straw. Once the waxhas hardened, remove it, trim off the excess wax and polish the scleral wax (Fig.6B). Waxscleral was re-tried to the patient to evaluate eye size, convexity, comfort, superior and inferior palpebral support and eye movement.

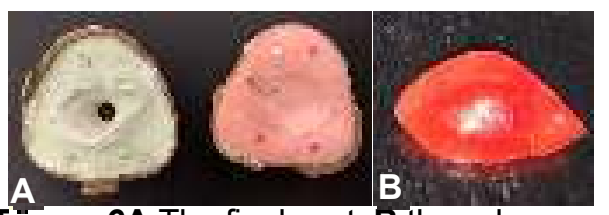

Fîgure 6A The final cast; $\mathrm{B}$ the sclera wax

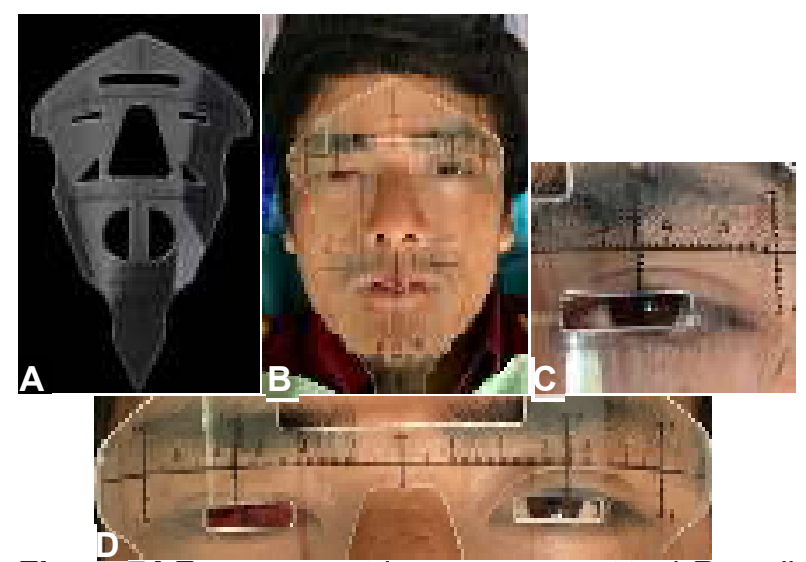

Figure 7AFace symmetric measurement tool; B application of the appliance to the patient's face; $\mathbf{C}$ measurement of iris and pupil diameters; $\mathbf{D}$ alignment

Determination of the position of the iris and pupil using a face symmetric measurement tool in the form of a face mask with vertical and horizontal line guidelines accompanied by centimeterand millimetersizes(Fig.7A). The patientwas instructed to sit up straight, with sclera wax in the patient's socket eye, applying a face symmetric measurement tool. Ensure the alignment of the vertical lines by connecting the trichion-glabella-subnasal-gnathion points so that they vertically divide the two sides of the face (Fig.7B).

Ensure the alignment of the 2 horizontal lines by connecting the left and right cheilon with the mesial-distal canthus of the right and left eyes. Then instruct the patient to look straight ahead, measure the diameter of the iris and pupil, mark the pupillary point with a permanentblackmarkeron the sclera wax (Fig.7C,D).

Coloring of the iris and sclera of the eye was performed with a camera taken in daylight at around ten o'clock in the morning (Fig.8A). The diameter of the iris is $11 \mathrm{~cm}$ which has been measured using a face symmetric measurement tool. Iris staining was carried out with a nylon brush no. 01 on a black plastic dick with a diameter of $11 \mathrm{~cm}$ with acrylic paint coloring with a mixture of burnt amber and rawamber colors in a 1:1 ratio. Before coloring the eyes, determine the midpoint of the plastic discand draw a diameter line from the vertical and horizontal.Staining starts from the inner ring out slowly and gently for an embossed effect, repeats after $30 \mathrm{mi}-$ nutes and applies black paint on the edges of the dick fora limbus effect. The plastic disc was left for 24 hours and then a 2-3 mm diameterblack plastic disc was attached as a pupil with multipurpose glue (Fig.8B).

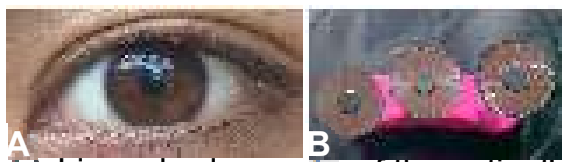

Figure $\mathbf{\delta} \mathbf{A}$ Iris and sclera coivur of the patient's eye; B iris staining on plastic discs.

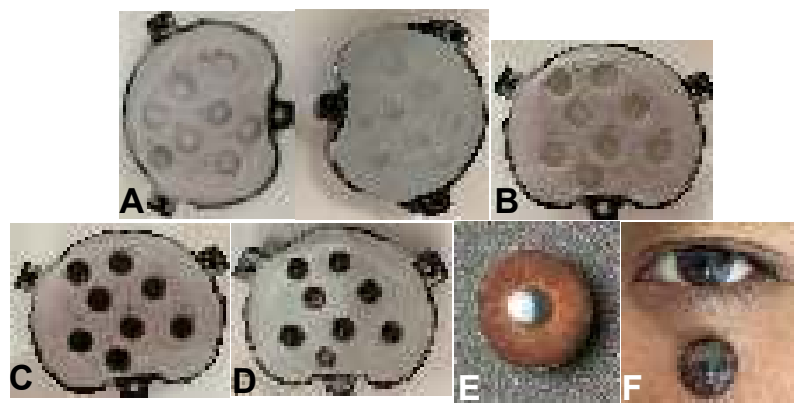

Figure 9A The eye doll mold; $B$ self cure clear acrylic on mold base; $\boldsymbol{C}$ laying the iris disc on self-cure clear acrylic; $\mathbf{D}$ the result of boiling the iris button; $\mathbf{E}$ iris button after finishing and polishing; $\mathbf{F}$ color of the iris button is similar to the real eye.

The iris button was made by duplicating the doll's eye and entered in a cuvette containing type III dental stone to obtain the iris button mold (Fig. 9A). Before to boiling, the molds were coated with CMS. The base of the mold is filled with self-cure clear acrylic(Fig.9B), wait for itto harden, then the iris is glued on top with multipurpose glue (Fig.9C). Apply heat-cured clear acrylic to the antagonist mold, closing, pressing and boiling. Open the results, do the finishing and polishing (Fig.9D,E,F).

Scleral wax which has been marked with a black point as a pupil, enlarged by $11 \mathrm{~mm}$. Remove the convexity of the eye on the wax sclera to the diameter of the iris to a depth of $2 \mathrm{~mm}$ and merge the iris button withoutany gaps between them (Fig. 10A). Try the scleral iris button in the patient and look at the alignment with the left eye with the use of the face symmetricmeasurement tool again (Fig. 10C,D). Re-instruct the patient to make eye movements; if the iris button is too convex, the convexity can be removed. Put the scleral wax back into the mold in the previous cuvette and coat with va- 
seline. To prevent the iris button from rotating when it is boiled, an acrylic cylindrical stick is attached to the top of the iris button withoutvaseline (Fig.10B). Open the previous antagonist cuvette model, refill it with type III dental stone.

Afterdewaxing (Fig.11A), apply CMS, fill in A3 color heatcure whiteacrylic, pressing and boiling . Open the results, with the stick still in a cuvette, do the finishing and polishing (Fig.11B).

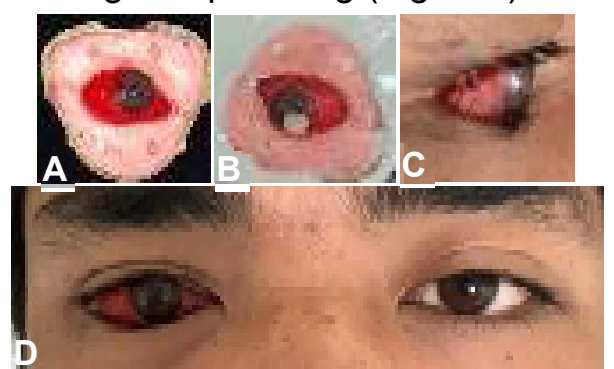

Figure 10A Merge of the iris button with sclera wax; $\mathbf{B}$ connect the stick with the iris button; $\mathbf{C}$ insertion of sclera wax and iris button from side view; $\mathbf{D}$ front view.

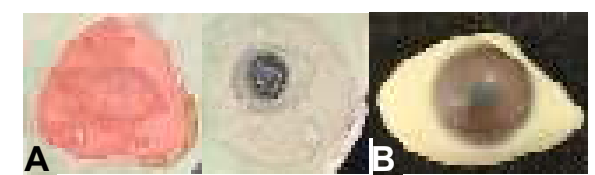

Figure 11A Dewaxing results; $B$ polished acrylic sclera.

The putty index (Fig.12A) as an indicator of depth is made by placing the putty at the bottom of the container and making a keyhole. Apply vaseline on the surface, fill the putty on the antagonist cap. Remove the surface of the acrylic sclera to a depth of $2 \mathrm{~mm}$ for subsequent clear acrylic application and measured using a putty index whose antagonist cap had been halved (Fig.12B,C). The iris button was finished and polished, except thesclera to application the scleral stain.

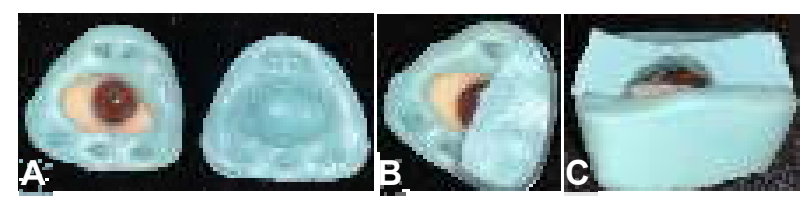

Figure 12A The putty index of $2 \mathrm{~mm}$ surface reduction; $\mathbf{B}$ cap of the putty index antagonist is halved; $\mathbf{C}$ indicator of scleral surface reduction

The color of the sclera was matched to that of the patient's eye (Fig.13A). The sclerawas stained with yellow, brown, and red colored pencils (Fig. 13B). The similarity of blood vessel fibers used red wool thread which was attached with monopoly syrup, waiting to dry (Fig.13C). Place the sclera back into the previous cuvette, apply CMS, apply heatcured clear acrylic, pressing and boiling (Fig.13D). Open the results, do the finishing and polishing (Fig.13E).

Before to insertion of the ocular prosthesis, the

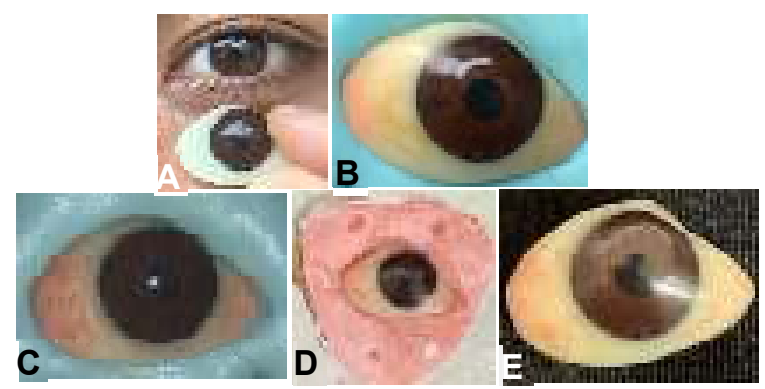

Figure 13A Color matching of the white acrylic sclera with the patient's eye; $\mathbf{B}$ scleral staining; $\mathbf{C}$ blood vessel fibers with red wool thread; $\mathbf{D}$ the acrylic sclera is returned to the cuvette for boiling; $\mathbf{E}$ ocular prosthesis.

eye socket was cleaned and ophthalmic fluid was applied. An ocular prosthesis was inserted from superior to inferior. Insertion of the ocular prosthesis by evaluating the size, convexity of the eye, comfort, superior and inferior palpebral support and eye movement (Fig.14A,B). If there is still something disturbing, it can be corrected by smoothing using sandpaper. Patient instruction and education on how to put on and take off the ocular prosthesis, how to clean and store it, and check-up visits.

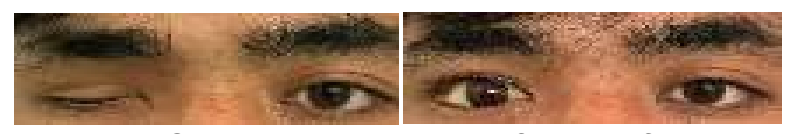

Figure 14 Ocular prostheses A before; $B$ after

\section{DISCUSSION}

Eye defects are maxillofacial deficienciesthat interfere with functional and social activities and require prostheticreplacement.Acustomocularprosthesis duplicates the orientation, color, contour, and size of the existing pupil and iris, providing realism and symmetry to the patient's face. The position of the iris is an important step and becomes a challenge in making ocularprostheses because symmetrical eyes provide good aesthetics so that it increases the patient's confidence. The literature shows many techniques for positioning the iris on ocularprosthesessuch as the use of a pupillometer, plastic strip template, millimeter ruler, ocular locator-fixed caliper, lightreflection symmetrical viewing of the eye, inverted anatomic tracings, Boley's gauge, transparent graph grid, computer simulation with optical scanning techniques. and computer-based design. ${ }^{6}$

Bhochhibhoya et al, used pupillary distance (PD) ruler in determining position of the iris which has a weakness in the case of facial asymmetry because this technique only uses the same guiding plane and cannot be used in cases of hypertelorism where both eyes cannot beaccommodated in the eye sockets of the PD ruler. ${ }^{2}$ Manjita et al, using glasses with added grids that can be continu- 
ed to the patient's facial skin has a weakness in preparation, which is the manufacture of custom scale grids which may lead to fabrication errors. ${ }^{3}$ Slightly differentfrom Lokhande using photographicassistance printed on self-adhesive glossy vinyl paper sticker attached to the glasses. ${ }^{6}$ Shetty et al, using the Hanau wide-view spring bow combined with a ruleralso has the disadvantage that clinicians must have a tool that is quite expensive and cannot be used in patients with ear loss. ${ }^{4}$ Similar to Ankita, using facebow combination frame with grid graph which has a weakness also in facial symmetry. ${ }^{7}$ In this paper, the author uses a face symmetric measurement tool to overcome these weaknesses.

Face symmetric measurement tool is a face mask that is usually used for eyebrow and lip tattoos. This tool has a guide of 1 vertical line and 2 horizontal lines accompanied by a ruler with units of centimeters-millimeters. These lines connect the symmetrical guide points of the face. The vertical line (Fig.15A) on this tool can divide the face symmetrically by drawing the trichion (tr)-glabella ( $g$ )subnasal (sn)-gnation (gn) line.

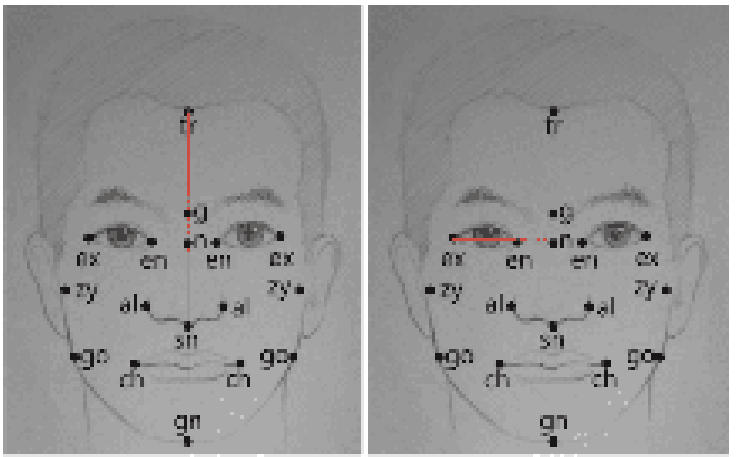

Figure 15A Symmetrical measuring guide points of vertical line faces: trichion (tr)-glabella (g)-subnasal (sn)gnation (gn); B symmetrical measurement guide points of the face of the upper horizontal line: mesial (en)-distal (ex) eye canthus and lower horizontal line: cheilon (ch). ${ }^{5}$

The first horizontal line (Fig.15B) is on the top, connecting the mesial (en)-distal (ex) points of the right and left eye canthus, while the second is on the bottom, connecting the left-right cheilon (ch) points. The first is used to measure the distance between the two pupils, the sizeand position of the pupil and iris. In this tool there is a rectangularhole in the eye that can easily mark the position of the pupil and iris directly into the sclera wax. This tool has ahandle on the bottom that is stable, comfort and easy for clinicians and patients to position it and can assess the iris position adequately.

The advantage sof using aface symmetric measurement tool are 1) a standard for measuring facial symmetry by connecting the guide points so that it can be used on asymmetrical faces;2) simple, economical, easy to use, does not require special skills, thereby reducing clinician's working time; 3 ) rigid, so it is stable, not easy to move in determining the point and can be used for right, left or both eyeloss;4) point determination can be done directly on the scleral wax because there is a square hole in the eye area; 5) a ruler with units of centimeters and millimeters, making it easy to measure the distance and size of the iris and pupil vertically and horizontally; 6) vertical and horizontal line guides making iteasy to divide the face and accurate location of the iris and pupil; 7) the handle under the chin can be held by the patient alone, comfortable without feeling tired or stiff, making it easier for clinicians to work; 8) transparent so you can see the guide points directly.

Theweakness of this tool is that this tool only has one size (free size) so that when the patient has a large reasonable width, the square hole in the tool cannotreach the location of the iris or pupil.

It is concluded that the use of the face symmetric measurement tool described here has given good results in terms of aesthetics, acceptability, and patient satisfaction. This tool is easy to use, does notrequire special skills, is accurate, stable, and can see facial symmetry compared to the techniques used previously.

\section{REFERENCES}

1. Guttal. A simple method of positioning the iris disk on a custom-made ocular prosthesis. a clinical report. JProsthodont 2008; 17: 223-7.

2. Bhochhibhoya. Alternative technique of iris orientation in a custom-made ocular prosthesis. J Prosthodont 2017:1-4

3. Manjita MP. Precise iris positioning in ocular prosthesis using an eyewear. J Dent Sci 2017; 2(1): 121.

4. Shetty PP. A iris positioning device and centering approach: A technique. J Prosthet Dent 2017.

5. Kim HJ. Heritabilities of facial measurements and their latent factors in Korean families. J Genomics \& Informatics 2013; 11(2).

6. Lokhande SP. Evaluation of patient acceptance using three techniques for the fabrication of ocular prosthesis: A case report. Int J Appl Dent Sci 2018; 4(3): 77-82

7. Ankita C. Iris positioning with a grid attached to a spring bow. J Clin Diagn Res 2017;11(3): 12-3 